\title{
Subquantum dark energy
}

\author{
Pedro F. González-Díaz \\ Centro de Física "Miguel A. Catalán," Instituto de Matemáticas y Física Fundamental, Consejo Superior de Investigaciones Científicas, \\ Serrano 121, 28006 Madrid, Spain \\ (Received 3 May 2003; published 20 May 2004)
}

\begin{abstract}
A procedure is considered that upgrades the Lagrangian description of quantum relativistic particles to the Lagrangian of a proper field theory in the case that the Klein-Gordon wave equation is classically interpreted in terms of a relativistic subquantum potential. We apply the resulting field theory to cosmology and show that the relativistic version of Bohm's subquantum potential which can be associated with a homogeneous and isotropic distribution of particles behaves as though it was a cosmological constant responsible for the current accelerating expansion of the Universe, at least in the limit where the field potential vanishes.
\end{abstract}

DOI: 10.1103/PhysRevD.69.103512

PACS number(s): 98.80.Cq, 98.80.Es

\section{INTRODUCTION}

With the advent of the increasingly compelling evidence for an accelerating expansion of the Universe [1-4], the traditional problem of the cosmological constant [5], which disturbed theoreticians a couple of decades ago, has now become even more acute [6], as it has actually been replaced by the problem of the nature of the so-called dark energy [7]. In scarcely five years a rather impressive influx of papers has been published which try to shed some light on the question of what type of stuff is homogeneously and isotropically pervading the Universe up to nearly $70 \%$ of its whole energy content and is able to currently make it evolve as though it was dominated by an antigravity regime [8]. Several candidates have been claimed to make up this dark energy. The first and most obvious option was-and still continues to be-a positive cosmological constant. The problem with this interpretation is similar to the old cosmological-constant problem: why is the currently required value of this quantity so much smaller than is predicted by fundamental theories [5]? Promoting the cosmological constant to the status of a vacuum field having its own dynamics has raised a rich plethora of possibilities which are described by means of a slowly varying scalar field generically denoted as either quintessence models [8] or tracking models [9], depending on whether the parameter $\omega$ entering the equation of state is or is not a constant. Recent constraints on that equation of state [10] seem nevertheless to point toward a value $\omega=-1$, which corresponds to the cosmological constant, or even [11] to values $\omega<-1$, which are associated with the so-called phantom dark-energy models that predict a big rip in finite time. Generalized Chaplygin gas models [12] and even further generalizations from them [13] have also been recently claimed to describe unified models of dark matter and energy. All such models have the interesting characteristic of describing by means of a single entity - the gasboth the dark matter and dark energy as given limiting cases. Finally, I would like to mention also the so-called tachyon model [14] for dark energy which quite remarkably constructs a concept of dark energy from very fundamental and simple relativistic principles which are promoted to their field-theory counterparts. Although there are many other interpretations of dark energy, a review of which can be found in Refs. $[7,8]$, those mentioned so far look the most promising.

In this paper we shall suggest a new interpretation for dark energy that is essentially based on a generalization of the tachyon model. Keeping in mind the idea that dark energy should somehow reflect the otherwise unobservable existence of a cosmological substance which will also have an essentially quantum-mechanical nature, and promoting the so-called Bohm classical interpretation of quantum mechanics [15] to the status of a field theory in a similar way to its development from classical relativistic mechanics to finally produce the model of tachyonic dark energy [15], we will thus be able to finally propose a simple "classical" fieldtheory model for dark energy which does not necessarily depend on the existence of any potential for the vacuum scalar field, and show the imprint of its truly quantum origin, formally in much the same way as does Bohm's classical interpretation of quantum mechanics.

Starting with an approximate approach, we develop some fundamental aspects of the field theory resulting from upgrading the "classically" interpreted quantum particle properties to field variables, which we also connect to current flat cosmology. Among the different limiting situations that we shall consider from that field theory, we single out one with vanishing field potential as the most interesting interpretation for dark energy in this paper: It is the subquantum potential associated with the particles that should be interpreted as being the cosmological constant responsible for dark energy.

The paper can be outlined as follows. In Sec. II we shall discuss a formalism based on applying some approximations to the relativistic energy-momentum relation with a subquantum potential. Fundamental aspects of the field theory are discussed in Sec. III where a recipe is given to compute the field potential. Section IV contains the description of a cosmological model which is based on coupling the full field Lagrangian to Hilbert-Einstein gravity. We summarize and conclude in Sec. V, where we also add some relevant discussion and comments.

\section{AN APPROXIMATE MODEL}

From the real part of the Klein-Gordon wave equation applied to a quasiclassical wave function $R \exp (i S / \hbar)$, where 
the probability amplitude $R\left(P=|R|^{2}\right)$ and the action $S$ are real functions of the relativistic coordinates, if the classical energy $E=\partial S / \partial t$ and momentum $p=\nabla S$ are defined, one can write [15]

$$
E^{2}-p^{2}+V_{S Q}^{2}=m_{0}^{2},
$$

where $m_{0}$ is the rest mass of the involved particle and $V_{S Q}$ is a relativistic subquantum potential,

$$
V_{S Q}^{2}=\frac{\hbar^{2}}{R}\left(\nabla^{2} R-\frac{\partial^{2} R}{\partial t^{2}}\right)
$$

which should be interpreted according to Bohm's idea [15] as the hidden subquantum potential that accounts for precisely defined unobservable relativistic variables whose effects would be physically manifested in terms of the indeterministic behavior shown by the given particles. From Eq. (2.1) it immediately follows that $p=\sqrt{E^{2}+V_{S Q}^{2}-m_{0}^{2}}$. Thus, since classically $p=\partial L / \partial[q(\dot{t})$ [with $L$ being the Lagrangian of the system and $q$ the spatial coordinates, which depend only on time $t, q \equiv q(t)]$, we have for the Lagrangian

$$
L=\int d \dot{q} p=\int d v \sqrt{\frac{m_{0}^{2}}{1-v^{2}}+M^{2}},
$$

in which $v=\dot{q}$ and $M^{2}=V_{S Q}^{2}-m_{0}^{2}$. The latter quantity is by no means ensured to be positive definite in the general theory. In fact, one could easily consider rest masses that would exceed the subquantum potential. Actually, if the particle is assumed to move locally according to some causal law, then the classical expression $E=\partial S / \partial t$ and $p=\nabla S$, where $S$ is the classical relativistic action, are locally satisfied. Hence, we can average Eq. (2.1) with a probability weighting function $P(x, t)=|R(x, t)|^{2}$, so that

$$
\begin{aligned}
\int & \iint d x^{3} P(x, t)\left(E^{2}-p^{2}+V_{S Q}^{2}\right) \\
= & \left\langle E^{2}\right\rangle_{\mathrm{av}}-\left\langle p^{2}\right\rangle_{\mathrm{av}}+\left\langle V_{S Q}^{2}\right\rangle_{\mathrm{av}}=m_{0}^{2},
\end{aligned}
$$

with the averaged quantities coinciding with the corresponding classical quantities and $\left\langle V_{S O}^{2}\right\rangle_{\text {av }}$ being a constant. Now the velocity of the particle can be defined to be

$$
\langle v\rangle_{\mathrm{av}}=\frac{\left\langle p^{2}\right\rangle_{\mathrm{av}}^{1 / 2}}{\left(\left\langle p^{2}\right\rangle_{\mathrm{av}}-\left\langle M^{2}\right\rangle_{\mathrm{av}}\right)^{1 / 2}},
$$

in which $\left\langle M^{2}\right\rangle_{\mathrm{av}}=\left\langle V_{S Q}^{2}\right\rangle_{\mathrm{av}}-m_{0}^{2}$. Thus, in the general particle theory, "effective" slower than light, faster than light, and lightlike particles can be defined to satisfy, respectively, $m_{0}>\left\langle V_{S Q}^{2}\right\rangle_{\mathrm{av}}, m_{0}<\left\langle V_{S Q}^{2}\right\rangle_{\mathrm{av}}$, and $m_{0}=\left\langle V_{S Q}^{2}\right\rangle_{\mathrm{av}}$, so implying different values and signs for the quantity $\left\langle M^{2}\right\rangle_{\mathrm{av}}$, and hence $M^{2}$.

In the classical limit $\hbar \rightarrow 0, V_{S Q} \rightarrow 0$, and hence we are left with just the classical relativistic Lagrangian for a particle with rest mass $m_{0}$. As shown by Bagla, Jassal, and Padmanabhan [14], promoting the quantities entering this simple Lagrangian to their field-theory counterparts allows us to get a cosmological model with tachyonic dark energy. In what remains of this section we shall explore the question of what kind of cosmological models can be derived if we apply an upgrading-to-field procedure starting with Lagrangian (2.3). For such an upgrading formalism we shall use throughout the paper the one employed by Padmanabhan et al. $[14,17,18]$, except for the harmless presence of the constant subquantum potential. Our procedure is thus based on upgrading the coordinate $q(t)$ to a field $\phi$ which, by relativistic invariance, will depend on both space and time while $\dot{q}^{2}$ is replaced by the quantity $\partial_{i} \phi \partial^{i} \phi$. This also makes it possible to regard the mass parameter $m_{0}$ as a potential function of the field $\phi$, thereby obtaining a given field theoretic Lagrangian. The Hamiltonian structure of the resulting theory will be algebraically similar to that of special relativity equipped with a subquantum potential term. The theory will in this way allow for solutions depending on both space and time, with finite momentum and energy densities analogous to some descriptions arising in string theory. For more details on that formalism and its motivation I address the reader to Refs. $[14,17,18]$.

Two approximate limiting situations will be considered in this section, starting with particle-theory cases where $m_{0}$ $\leqslant V_{S Q}$ which ensures that $M^{2}>0$. First of all, we shall look at the case of most cosmological interest, which corresponds to the limit of small values of the rest mass, $m_{0} \rightarrow 0$, for which the Lagrangian becomes

$$
\begin{aligned}
L & \simeq \sqrt{V_{S Q}^{2}-m_{0}^{2}} \int d v\left(1+\frac{m_{0}^{2}}{2\left(V_{S Q}^{2}-m_{0}^{2}\right)\left(1-v^{2}\right)}\right) \\
& =\sqrt{V_{S Q}^{2}-m_{0}^{2}} v+\frac{m_{0}^{2}}{\sqrt{V_{S Q}^{2}-m_{0}^{2}}} \ln \left[\left(\frac{1+v}{1-v}\right)^{1 / 4}\right] .
\end{aligned}
$$

This Lagrangian is positive definite whenever $V_{S Q}>0$. For nonzero values of the subquantum potential, we can have physical systems with nonzero Lagrangian even for the massless case where $v=1$ and $m_{0}=0$ simultaneously. This is made possible since the existence of the subquantum potential allows us to consider an "effective" rest mass given by $M \equiv \sqrt{V_{S Q}^{2}-m_{0}^{2}}$. On the other hand, since the subquantum potential $V_{S Q}$ can take on both positive and negative values, the associated field theory can lead to positive or negative pressure, respectively. Choosing $V_{S Q}<0$ and hence $L<0$, in the massless case $m_{0}=0, v=1$, we have

$$
L=-\left|V_{S Q}\right| .
$$

Generalizing to a field theory in the general case $m_{0} \neq 0, v$ $<1$ requires the upgrading $q(t) \rightarrow \phi$, a field which will thereby depend on both space and time, $\phi(r, t)$, replacing $v^{2} \equiv \dot{q}^{2}$ by $\partial_{i} \phi \partial^{i} \phi$ and the rest mass $m_{0}$ by a generic potential $V(\phi)$. In the extreme massless case, however, the Lagrangian (2.5) does not contain any quantity that can be up- 
graded to depend on $\phi$, so that the Lagrangian for the field theory in the massless case is no longer zero, but it is also given by Eq. (2.5).

We shall regard here the Lagrangian (2.5) as containing all the cosmological information that corresponds to a universe whose dark energy is given by a positive cosmological constant, provided the field $\phi$ is homogeneously and isotropically distributed. This can be accomplished if, e.g., the subquantum potential is interpreted as that potential associated with the hidden dynamics of the particles, which are homogeneously and isotropically distributed in the universe. Assuming next a perfect fluid form for the equation of state of the cosmic field $\phi$, i.e., introducing a stress-energy tensor

$$
T_{k}^{i}=(\rho+P) u^{i} u_{k}-p \delta_{k}^{i},
$$

where the energy density $\rho$ and the pressure $p$ that correspond to Lagrangian (2.5) are given by

$$
\rho=\left|V_{S Q}\right|, \quad p=-\left|V_{S Q}\right|,
$$

and the four-velocity is

$$
u_{k}=\frac{\partial_{k} \phi}{\sqrt{\partial_{i} \phi \partial^{i} \phi}} .
$$

From Eqs. (2.7) and the conservation equation for cosmic energy, $d \rho=-3(\rho+p) d a / a$, it again follows that $\rho=\kappa^{2}$ $=\left|V_{S Q}\right|=$ const, so that the resulting Friedmann equation $\dot{a}$ $=\kappa a / m_{P}\left(m_{P}\right.$ being the Planck mass $)$ yields the expected solution for the scale factor $a=a_{0} \exp \left[\kappa\left(t-t_{0}\right) / m_{P}\right]$. Equations (2.7) immediately lead, moreover, to a characteristic parameter for the perfect fluid state equation which turns out to be constant and given by $\omega=P / \rho=-1$. We can conclude therefore that if $m_{0}=0, v=1$ [i.e., $V(\phi)=0$ and $\partial_{i} \phi \partial^{i} \phi$ $=1$ in the field theory] and $V_{S Q}<0$, the hidden dynamics of particles or fields causes a subquantum potential to appear, inducing the presence of a pure cosmological constant given by $\Lambda=\kappa=\sqrt{V_{S Q}}$. When the rest mass is $m_{0} \neq 0$ and very small, there would be a nonzero field-theory potential $V(\phi) \rightarrow m_{0}$ and the subquantum medium would correspond to a cosmic dark energy which would somehow behave like some sort of "tracking" quintessential field [9]. In fact, in such a case we had for negative $V_{S Q}$ and small but nonzero $m_{0}$,

$$
L=p=-|M| \sqrt{\partial_{i} \phi \partial^{i} \phi}-\frac{V(\phi)^{2}}{4|M|} \ln \left(\frac{1+\sqrt{\partial_{i} \phi \partial^{i} \phi}}{1-\sqrt{\partial_{i} \phi \partial^{i} \phi}}\right),
$$

with $M$ being now given by $M \equiv M[V(\phi)]$ $=-\sqrt{V_{S Q}^{2}-V(\phi)^{2}}$, where $V(\phi)$ is again generally defined through the upgrading procedure, i.e., $m_{0} \rightarrow V(\phi)$. The pressure $p$ is then a definite negative quantity such that $\partial_{i} \phi \partial^{i} \phi$ $<2 V(\phi)$ only if $\partial_{i} \phi \partial^{i} \phi$ is sufficiently smaller than $\left(\partial_{i} \phi \partial^{i} \phi\right)_{c}$, with

$$
\frac{\sqrt{\left(\partial_{i} \phi \partial^{i} \phi\right)_{c}}}{1-\left(\partial_{i} \phi \partial^{i} \phi\right)_{c}}=\ln \left[\frac{1+\sqrt{\left(\partial_{i} \phi \partial^{i} \phi\right)_{c}}}{\sqrt{1-\left(\partial_{i} \phi \partial^{i} \phi\right)_{c}}}\right] .
$$

The energy density which together with the pressure $p$ enters the equation of state $p=\omega(\phi) \rho$ would then read

$$
\rho=-\frac{V(\phi)^{2}}{2|M(\phi)|}\left[\frac{\sqrt{\partial_{i} \phi \partial^{i} \phi}}{1-\partial_{i} \phi \partial^{i} \phi}-\ln \left(\frac{1+\sqrt{\partial_{i} \phi \partial^{i} \phi}}{\sqrt{1-\partial_{i} \phi \partial^{i} \phi}}\right)\right] .
$$

We then note that for the considered range of the kinetic term we can always in fact choose a range for the parameter entering the equation of state which satisfies $0 \geqslant \omega(\phi) \geqslant-1$.

In the limit that the rest mass and the subquantum potential take on very similar values, which is the second situation we shall briefly consider, the Lagrangian can be approximated as

$$
L \simeq m_{0} \int \frac{d v}{\sqrt{1-v^{2}}}=\frac{1}{2} m_{0} \ln \left(\frac{1-\sqrt{1-v^{2}}}{1+\sqrt{1-v^{2}}}\right)^{1 / 2} .
$$

Such a Lagrangian is negative definite and, if we upgrade the quantities involved in it so that they become field-theory variables, $m_{0} \rightarrow V(\phi)$, with $V(\phi)$ a classical potential for the scalar field $\phi$, and $v^{2} \rightarrow \partial_{i} \phi \partial^{i} \phi$, it corresponds to a negative pressure

$$
p=\frac{1}{2} V(\phi) \ln \left(\frac{1-\sqrt{1-\partial_{i} \phi \partial^{i} \phi}}{1+\sqrt{1-\partial_{i} \phi \partial^{i} \phi}}\right),
$$

which is definite negative, and a positive energy density

$$
\rho=\frac{V(\phi)}{\sqrt{1-\partial_{i} \phi \partial^{i} \phi}}-p .
$$

Thus, for a perfect fluid equation of state $p=\omega(\phi) \rho$, this would again be somehow analogous to a tracking quintessencelike field. Anyway, the simplest and most interesting situation we have dealt with in this section corresponds to the massless case where the consideration of the particles in terms of the relativistic version of the classical Bohm's theory is enough to promote their corresponding subquantum potential to the same status as that of a cosmological constant. Therefore, if we adopt the interpretation considered in this article, dark energy appears to at least partly correspond to the overall work which is done by all particles along their hidden trajectories associated through the Bohm interpretation with the essential quantum indeterminacy of the observable matter in the Universe.

\section{THE FIELD THEORY}

In order to construct a field theory starting with the classical relation $E^{2}-p^{2}+V_{S Q}^{2}=m_{0}^{2}$, one should first integrate Eq. (2.3) to yield the Lagrangian in closed form,

$$
L=-m_{0} E(x, k),
$$


where $E(x, k)$ is the elliptic integral of the second kind [16], with

$$
x=\arcsin \sqrt{1-v^{2}}, \quad k=\sqrt{1-\frac{V_{S Q}^{2}}{m_{0}^{2}}} .
$$

The Lagrangian (3.1) describes a relativistic particle with a (one-dimensional) position $q(t)$ and a mass $m_{0}$ whose local motion is causally disturbed by the presence of a subquantum potential $V_{S Q}$. One can now proceed to upgrading [14] the particle theory into a field theory by promoting $m_{0}$ $\rightarrow V(\phi)$ and $v^{2} \rightarrow \partial^{i} \phi \partial_{i} \phi$ in Lagrangian (3.1). Thus, we obtain

$$
L=-V(\phi) E(x(\phi), k(\phi))
$$

in which

$$
x(\phi)=\arcsin \sqrt{1-\partial^{i} \phi \partial_{i} \phi}, \quad k(\phi)=\sqrt{1-\frac{V_{S Q}^{2}}{V(\phi)^{2}}} .
$$

We shall restrict ourselves in Secs. III and IV to considering the field-theoretic analogue to "effective" slower than light relativistic particles filling a flat universe with the conventional scalar field $\phi$ having the potential $V(\phi)$ as a source. The evolution of that universe will be assumed to be specified so that the scale factor $a(t)$ and its time derivatives $H(t) \equiv(\dot{a} / a), \ldots$ are all known functions of time $t$. For a Friedmann-Robertson-Walker (FRW) universe $\phi(t, \mathbf{x})$ $=\phi(t)$. Thus, our problem will be to determine $\phi$ and hence the potential for the scalar field $V(\phi)$ as given functions of the specified cosmic parameters $H(t)$ and $\dot{H}(t)$.

The Friedmann equations are

$$
H^{2}=\frac{8 \pi G}{3} \rho_{T}, \quad \frac{\ddot{a}}{a}=-\frac{4 \pi G}{3}\left(\rho_{T}+3 p_{T}\right),
$$

with $\rho_{T}=\rho_{o b}+\rho_{\phi}$ the energy density for observable matter plus dark energy, and $p_{T}$ the corresponding sum of pressures. For a theory that generalizes the nonrelativistic description of a single particle, that is, for a typical quintessence theory, the field $\phi(t)$ is defined by the conventional expressions

$$
\rho_{\phi}=\frac{1}{2} \dot{\phi}^{2}+V(\phi), \quad p_{\phi}=\frac{1}{2} \dot{\phi}^{2}-V(\phi) .
$$

For the FRW universe the Lagrangian generalizing the relativistic particle Lagrangian plus a subquantum potential reduces to Eq. (3.3) with

$$
x(\dot{\phi})=\arcsin \sqrt{1-\dot{\phi}^{2}}, \quad k(\phi)=\sqrt{1-\frac{V_{S Q}^{2}}{V(\phi(t))^{2}}} .
$$

In the limit $V_{S Q} \rightarrow 0$, that Lagrangian becomes the Lagrangian for the tachyon model $[14,17,18]$, i.e., $L=-V(\phi) \sqrt{1-\dot{\phi}^{2}}$, as should be expected. The pressure and energy density for our field model to be used in Eq. (3.5) are no longer given by Eqs. (3.6), but the new "relativistic" expressions

$$
p_{\phi}=-V(\phi) E(x, k)
$$

$$
\rho_{\phi}=\frac{V(\phi) \sqrt{1-\left[\Delta V^{2}\left(1-\dot{\phi}^{2}\right) / V(\phi)^{2}\right]} \dot{\phi}}{\sqrt{1-\dot{\phi}^{2}}}+V(\phi) E(x, k),
$$

where $\Delta V^{2}=V(\phi)^{2}-V_{S Q}^{2}$. Notice that (i) in the limit $V_{S Q} \rightarrow 0 \quad \rho_{\phi} \rightarrow V(\phi) / \sqrt{1-\dot{\phi}^{2}}$ and $p_{\phi} \rightarrow V(\phi) \sqrt{1-\dot{\phi}^{2}}$, i.e., expressions that respectively correspond to the energy density and pressure in the tachyon model $[14,17,18]$; and (ii) the state-equation parameter $\omega(t)=p_{\phi} / \rho_{\phi}$ derived from Eqs. (3.8) and (3.9) is generally larger than -1 and is therefore associated with a dark-energy content which does not match a cosmological constant. In any case, for a source with parameter $\omega(t)=p_{\phi} / \rho_{\phi}$, we must always have [17]

$$
\frac{\dot{\rho}_{\phi}}{\rho_{\phi}}=-3 H(1+\omega)=\frac{2 \dot{H}}{H} .
$$

Combining Eq. (3.10) with the expression for $\omega(t)$, we obtain

$$
\frac{\sqrt{1-\left[\Delta V^{2}\left(1-\dot{\phi}^{2}\right) / V(\phi)^{2}\right]} \dot{\phi}}{\sqrt{1-\left[\Delta V^{2}\left(1-\dot{\phi}^{2}\right) / V(\phi)^{2}\right]} \dot{\phi}+E(x, k) \sqrt{1-\dot{\phi}^{2}}}=-\frac{2 \dot{H}}{3 H^{2}} .
$$

On the other hand, multiplying Eqs. (3.8) and (3.9) and using Eq. (3.10) and the Friedmann equation, one can derive

$$
V(\phi)=\frac{\left(3 H^{2} / 8 \pi G\right)\left(1+2 \dot{H} / 3 H^{2}\right)^{1 / 2}}{\left\{\left[\frac{\sqrt{1-\left[\Delta V^{2}\left(1-\dot{\phi}^{2}\right) / V(\phi)^{2}\right]} \dot{\phi}}{\sqrt{1-\dot{\phi}^{2}}}+E(x, k)\right] E(x, k)\right\}^{1 / 2}},
$$


where

$$
E(x, k)=-\sqrt{\frac{1-\Delta V^{2}\left(1-\dot{\phi}^{2}\right) / V(\phi)^{2}}{1-\dot{\phi}^{2}}} \dot{\phi}\left(1+\frac{3 H^{2}}{2 \dot{H}}\right) .
$$

Equations (3.11) and (3.12) would solve the problem posed initially, that is, the problem of obtaining the field potential in terms of the scalar field $V(\phi)$ and the scalar field in terms of time $\phi(t)$. In fact, from the above equations we can obtain a simpler expression for $V(\dot{\phi})$ which is given by

$$
V(\dot{\phi})=-\left[\left(\frac{2 \dot{H}}{8 \pi G}\right)^{2}-\dot{\phi}^{2} V_{S Q}^{2}\right] \frac{\sqrt{1-\dot{\phi}^{2}}}{\dot{\phi}^{2}} .
$$

Now, by inserting $V(\dot{\phi})$ into Eq. (3.11) and integrating the resulting expression over time $t$ one would attain an expression for $\phi(t)$. Reexpressing $\phi(t)$ then as a function of $\dot{\phi}(t)$ and the given scale factor $a(t)$, an expression for $V(\phi)$ compatible with the original hypothesis could finally be obtained. In principle, given any scale factor $a(t)$, one can then obtain $V(t)$ and $\phi(t)$, and hence the potential $V(\phi)$ by using Eqs. (3.11), (3.12), and (3.14). However, these equations are very complicated and cannot be immediately solved except for certain limiting approximated cases. In what follows of this section, we shall consider two such limiting cases where these equations can be easily solved. First, if we let $V_{S O}$ $\rightarrow 0$, we immediately recover the tachyon-model expression which was already dealt with by Padmanabhan [17]. If, on the other hand, one lets $V(\phi)^{2} \rightarrow V_{S Q}^{2}$, then we can derive the approximate expressions

$$
\begin{gathered}
\frac{\dot{\phi}}{\dot{\phi}+\sqrt{1-\dot{\phi}^{2}} \arcsin \sqrt{1-\dot{\phi}^{2}}} \simeq-\frac{2 \dot{H}}{3 H^{2}}, \\
V \simeq \frac{\left(3 H^{2} / 8 \pi G\right)\left(1+2 \dot{H} / 3 H^{2}\right)^{1 / 2}\left(1-\dot{\phi}^{2}\right)^{1 / 4}}{\sqrt{\left(\dot{\phi}+\sqrt{1-\dot{\phi}^{2}} \arcsin \sqrt{1-\dot{\phi}^{2}}\right) \arcsin \sqrt{1-\dot{\phi}^{2}}}} .
\end{gathered}
$$

Restricting ourselves then to the late-time regime of slowly varying field, $\dot{\phi} \rightarrow 0$, and nearly constant $H$, we finally get

$$
\phi \simeq-\pi \int \frac{\dot{H} d t}{3 H^{2}}, \quad V \simeq \frac{3 H^{2}}{4 \pi^{2} G}\left(1+\frac{2 \dot{H}}{3 H^{2}}\right) \rightarrow V_{S Q} .
$$

These values of $\phi$ and $V \simeq V_{S O}$ are very similar indeed to those of the tachyon model $[14,17,18]$. They are in fact approximately the same except for a factor $\pi / 2$ in $\phi$ and a factor $2 / \pi$ in $V(t)$. If we finally consider a late universe with power law expansion $a=t^{n}$, then we get $\phi \simeq \pi t / 3 n+\phi_{0}$, which results in a potential $V(\phi) \simeq 3 /\left[12 G\left(\phi-\phi_{0}\right)^{2}\right]$.

\section{A COSMOLOGICAL MODEL}

Let us consider in this section the observationally most favored case of a spatially flat universe with Friedmann equations given by Eqs. (3.5). For our scalar field we have an energy density and a pressure as given in Eqs. (3.8) and (3.9), respectively. Writing the stress tensor in the perfect fluid form shown by Eq. (2.6) and four-velocity $u_{k}$ as expressed in Eq. (2.8), we can now uncover that the stress tensor for the field $\phi$ may be regarded as being the sum of a pressureless dust component plus a vacuum cosmologicalconstant-like component. This can most clearly be seen by breaking up the energy density $\rho_{\phi}$ and pressure $p_{\phi}$ as $[14,18]: \rho_{\phi}=\rho_{v}+\rho_{D M}, p_{\phi}=p_{v}+p_{D M}$, with

$$
\rho_{v}=V(\phi) E(x, k), \quad \rho_{D M}=\frac{V(\phi)}{\sqrt{1-\dot{\phi}^{2}}} \sqrt{1-\frac{\Delta V^{2}\left(1-\dot{\phi}^{2}\right)}{V(\phi)^{2}}}
$$

$p_{v}=-V(\phi) E(x, k), \quad p_{D M}=0$,

where we note that $\omega_{v}=p_{v} / \rho_{v}=-1$, so that dark energy behaves like a cosmological constant.

The present description resembles that of generalized Chaplygin gas models [12], which likewise describe dark matter and pure dark energy as the extreme limiting cases for a single field at the highest and smallest densities, respectively. In the present case, the choice in Eqs. (4.1) and (4.2) allows us to write for early time

$$
\lim _{t \rightarrow 0}[V(\phi) E(x, k)] \rightarrow 0
$$

and therefore $\dot{\phi} \rightarrow \pm 1$ as $t \rightarrow 0$. On the other hand, for large $t$,

$$
\lim _{t \rightarrow \infty}\left[\frac{V(\phi)}{\sqrt{1-\dot{\phi}^{2}}} \sqrt{1-\frac{\Delta V^{2}\left(1-\dot{\phi}^{2}\right)}{V(\phi)^{2}}}\right] \rightarrow 0
$$

which in turn implies that for $V(\phi) \neq 0, \quad \Delta V^{2}(1$ $\left.-\dot{\phi}^{2}\right) / V(\phi)^{2} \rightarrow 1$; that is, if for example $V(\phi)^{2} \gg V_{S Q}$, then $\dot{\phi} \rightarrow 0$, thus matching the classical, slowly varying behavior of a scalar field that characterizes quintessential models.

On the other hand, the action that couples the scalar field $\phi$ and the given observable matter fields to Hilbert-Einstein gravity can be written as

$$
S=\int d^{4} x \sqrt{-g}\left[\frac{R}{16 \pi G}-V(\phi) E(x, k)+L_{\mathrm{ob}}\right],
$$

where $L_{\mathrm{ob}}$ is the Lagrangian for the observable matter field we should add to dark matter and energy in order to represent a realistic universe. The latter nongravitational components, i.e., dark matter and dark energy, are both unitarily described by just the scalar field $\phi$. We can now derive the equation of motion for such a field in our cosmological context to be 


$$
\begin{aligned}
\dot{\phi} \ddot{\phi}= & -\left(1-\dot{\phi}^{2}\right) \sqrt{1-\left(1-\dot{\phi}^{2}\right)\left(1-\frac{V_{S Q}^{2}}{V(\phi)^{2}}\right)} \\
& \times\left[3 H \sqrt{1-\left(1-\dot{\phi}^{2}\right)\left(1-\frac{V_{S Q}^{2}}{V(\phi)^{2}}\right)}\right. \\
& +\sqrt{1-\dot{\phi}^{2}}\left(\frac{d V(\phi)}{V(\phi) d \phi}\right) \\
& \left.\times\left(\frac{E(x, k) V(\phi)^{2}-F(x, k) V_{S Q}^{2}}{V(\phi)^{2}-V_{S Q}^{2}}\right)\right],
\end{aligned}
$$

where

$$
F(x, k)=F\left(\arcsin \sqrt{1-\dot{\phi}^{2}}, \sqrt{1-\frac{V_{S Q}^{2}}{V(\phi)^{2}}}\right)
$$

is the elliptic integral of the first kind [16], with the same argument $x$ and parameter $k$ as the integral $E(x, k)$.

Inspection of Eq. (4.6) leads us to notice that, as happens in the tachyon theory $[14,17,18]$, the change of $\dot{\phi}$ goes to zero as it approaches \pm 1 or, which is specific to the present model, $\pm V_{S Q} /\left[V(\phi) \sqrt{V_{S Q}^{2} / V(\phi)^{2}-1}\right]$. In these cases the equation of state for the field $\phi$ is dustlike. Thus, at any stage, if the field behaves like dust, it continues to do so for a long time. Such a behavior persists for a duration that depends on the closeness of $\dot{\phi}$ to \pm 1 or $\pm V_{S Q} /\left[V(\phi) \sqrt{V_{S Q}^{2} / V(\phi)^{2}-1}\right]$. The detailed behavior of the field evolution would depend on the shape of the potential used. On the other hand, the change of $\dot{\phi}$ goes to infinity as it approaches zero. In that case, it can be checked that the energy density $\rho_{v}=-p_{v}$ should be given in terms of the complete elliptic integral of the second kind [16], $E(k$ $\left.=\sqrt{1-V_{S Q}^{2} / V(\phi)^{2}}\right)$, while $\rho_{D M}$ is given by $V_{S Q}$.

Even for the simplest possible potentials, the solutions to Eq. (4.6) and the associated Friedmann equation is rather a cumbersome task. Numerical calculations are left for future work.

\section{SUMMARY AND DISCUSSION}

In this paper we have developed the essential, preliminary aspects of a new field theory and its application to cosmology. It is based on the novel idea that vacuum fields can correspond to an upgrading of the Lagrangian of the quantum relativistic description of a single particle, as interpreted in terms of the "classical" subquantum potential, to a scalar field theory. Some rather heuristic arguments were first advanced that led to the interpretation that the field-theory Lagrangian approximately becomes the subquantum potential in the limit of small values of the field potential and the largest values of the kinetic term. In what follows we shall comment on this reduction process from which we can deduce that the equation of state for dark energy at that limit is $p=-\rho$; i.e., the equation of state for a pure cosmological constant whose dynamics is fully hidden. A brief classical description of the full field theory is then carried out. This leads us to formulate the way in which the potential for the scalar field can be obtained in a cosmological context where the universe is spatially flat. Some cosmic predictions are discussed afterward based on the formulation of the equation of motion for the scalar field.

We shall now consider a couple of limiting cases from the full field theory developed in Secs. III and IV. We first derive the approximate form of the Lagrangian when we let $V(\phi)$ $\rightarrow 0, \dot{\phi} \rightarrow \pm 1$, keeping $V_{S Q}$ nonzero. This case was already considered starting directly from an approximate expression for the classical momentum by using heuristic arguments. We shall check that the results obtained in Sec. II are again obtained by using Eq. (3.1). In fact, if $V_{S Q}^{2} \gg V(\phi)^{2}$, the Lagrangian in Eq. (3.1) can be written as

$$
\begin{aligned}
L= & -V_{S Q}\left[E\left(\frac{V_{S Q}}{V(\phi)} x, \kappa\right)\right. \\
& \left.+\kappa \operatorname{sn}\left(\sqrt{1-\dot{\phi}^{2}} \frac{V_{S Q}}{V(\phi)}, \kappa\right) \mathrm{cd}\left(\sqrt{1-\dot{\phi}^{2}} \frac{V_{S Q}}{V(\phi)}, \kappa\right)\right],
\end{aligned}
$$

where

$$
\kappa=\sqrt{1-\frac{V(\phi)^{2}}{V_{S Q}^{2}}}
$$

and sn and cd are elliptic functions [16]. Now, in the limit $\sqrt{1-\dot{\phi}^{2}} \rightarrow 0$ and $V(\phi) \rightarrow 0$ the Lagrangian (5.1) reduces to

$$
L \simeq-V_{S Q}\left[\sin \left(\frac{\sqrt{1-\dot{\phi}^{2}} V_{S Q}}{V(\phi)}\right)-\tanh \left(\frac{\sqrt{1-\dot{\phi}^{2}} V_{S Q}}{V(\phi)}\right)\right],
$$

which can be written as $L \sim-V_{S Q}$ since $\sqrt{1-\dot{\phi}^{2}} / V(\phi)$ is an indeterminate quantity. Consistency of this and the results to follow is ensured by checking that, in fact, as one lets $V_{S Q} \rightarrow 0$ the Lagrangian of the tachyon theory $[14,17,18]$, $L=-V(\phi) \sqrt{1-\dot{\phi}^{2}}$, is recovered from Eq. (3.1). The result in Eq. (5.2) can again be derived in the limit where $V(\phi)$ $\rightarrow V_{S Q}$; that is,

$$
L \simeq-V_{S Q} \arcsin \sqrt{1-\dot{\phi}^{2}},
$$

if one considers a slowly varying field such that $\dot{\phi} \rightarrow 0$. Thus, we again get $L \simeq-V_{S Q}$ and hence once again $p \simeq-V_{S Q}$, $\rho \simeq V_{S Q}$, corresponding to a cosmological constant with state equation parameter $\omega=-1$. If, instead of $\dot{\phi} \rightarrow 0$, we had taken $\dot{\phi} \rightarrow \pm 1$, then a new field theory similar to tachyon theory but with the potential $V(\phi)$ replaced with the subquantum potential $V_{S Q}$ would have been obtained.

Consider finally the hypothetical case where $\dot{\phi}^{2}>1$. If we then rotate the field-theory potential $V(\phi)$ to purely imaginary values so that $V(\phi) \rightarrow i W(\phi)$, then the Lagrangian takes the form 


$$
\begin{aligned}
L(\phi)= & -W(\phi)[E(y, \gamma)-F(y, \gamma) \\
& \left.-\sqrt{\dot{\phi}^{2}-1}\left(1+\frac{\left(\dot{\phi}^{2}-1\right) V_{S Q}^{2}}{\dot{\phi}^{2} W(\phi)^{2}}\right)\right],
\end{aligned}
$$

with

$$
y=\arctan \sqrt{\dot{\phi}^{2}-1}, \quad \gamma=\frac{V_{S Q}}{V(\phi)},
$$

and $F(y, \gamma)$ again being the elliptic integral of the first kind. Clearly, in the limit $V_{S Q} \rightarrow 0$, we get what one could denote as a tachyon field theory from tachyonic particles for which

$$
L \simeq W(\phi) \sqrt{\dot{\phi}^{2}-1},
$$

and hence

$$
p \simeq W(\phi) \sqrt{\dot{\phi}^{2}-1}, \quad \rho \simeq \frac{W(\phi)}{\sqrt{\dot{\phi}^{2}-1}} .
$$

Note that in this case the pressure associated with the scalar field is positive as that field must now be a superluminarly varying field and therefore $\dot{\phi}^{2} / 2>W(\phi)$.

Before closing, I will add some comments which may be helpful for a better understanding of some points of this paper. First of all, we ought to notice that, in addition to the limiting case where $v \rightarrow \pm 1$ and $m_{0} \rightarrow 0$, one should apply the Bohm interpretation and the upgrading procedure to vacuum massive particles. In that case, too, the existence of a positive pressure making the universe viable may be ensured by providing in a quite natural way the observable Lagrangian $L_{\mathrm{ob}}$ in the action (4.5) with a suitable equation of state. A mutual balance between such a positive pressure and the negative pressure $p_{\phi}$ would naturally arise as a consequence from the comparison between the equations of motion for the observable matter fields and Eqs. (4.1), (4.2), and (4.6). What one would conventionally expect from that comparison is that, after passing through a pressureless regime with $\phi$ dark matter at the earliest universe, this would become dominated first by a positive-pressure regime with observable energy and then by the negative-pressure late darkenergy $\phi$ regime after reaching the coincidence time [19].

In principle, there are two possible interpretations for field $\phi$. On the one hand, $\phi$ could be viewed as the "classical" field which arises from the hidden dynamics that led to the apparent quantum behavior of all particles and cosmic fields, including the cosmic microwave background. If such an interpretation is accepted, then there would not be anything like dark energy. On the other hand, the field $\phi$ could also be regarded as making up the real stuff for the existing dark energy. Although the former interpretation might perhaps be accommodated in the limiting case where $\dot{\phi}= \pm 1, V(\phi)$ $=0$, the latter view appears to be more general and convenient.

In the general case where $V(\phi) \neq 0$, obviously the evolution of the universe cannot be dissociated from $V(\phi)$. Thus, the general and limiting models discussed in this paper can all be tested by following the evolution of the energy density and pressure that is predicted by specifying $V(\phi)$ according to Eqs. (3.11) and (3.12) for the general case, or to limiting expressions such as, e.g., those in Eqs. (3.14). Determining the potential $V(\phi)$ in this way will allow us to check, moreover, the extent to which the models with $\dot{\phi} \neq \pm 1$ of Sec. II share some characteristics of tracking quintessence fields. It remains an open question, however, to see whether or not the general model suggested in this paper might add to a possible solution to the coincidence time problem [19].

It is finally noted that, even though a model involving a modification of the relativistic point particle quantum energy-momentum relation and its conversion into a classical field theory relationship looks more complicated than other models for dark energy, it contains some of those simpler models as limiting cases and shows a much wider applicability.

\section{ACKNOWLEDGMENTS}

The author thanks Carmen L. Sigüenza for useful discussions. This work was supported by DGICYT under Research Project BMF2002-03758.
[1] S. Perlmutter et al., Astrophys. J. 483, 565 (1997); Supernova Cosmology Project, S. Perlmutter et al., Nature (London) 391, 51 (1998); P.M. Garnavich et al., Astrophys. J. Lett. 493, L53 (1998); B.P. Schmidt, Astrophys. J. 507, 46 (1998); A.G. Riess et al., ibid. 116, 1009 (1998).

[2] M.S. Turner, "Dark energy and the new cosmology," contribution to the SNAP Yellow Book, Snowmass, 2001, astro-ph/0108103.

[3] A. Riess et al., Astrophys. J. 560, 49 (2001).

[4] M. Carmeli, "Accelerating Universe, Cosmological Constant and Dark Energy, astro-ph/0111259.

[5] S. Weinberg, Rev. Mod. Phys. 61, 1 (1989).

[6] S.L. Bridle, O. Lahav, J.P. Ostriker, and P.J. Steinhardt,
Science 299, 1532 (2003); T. Padmanabhan, Phys. Rep. 380, 235 (2003).

[7] For reviews and an extensive collection of papers on dark energy, see the website www.to.infn giunti/NU/Cosmology/

[8] C. Wetterich, Nucl. Phys. B302, 668 (1988); J.C. Jackson and M. Dodgson, Mon. Not. R. Astron. Soc. 297, 923 (1998); J.C. Jackson, ibid. 296, 619 (1998); R.R. Caldwell, R. Dave, and P.J. Steinhardt, Phys. Rev. Lett. 80, 1582 (1998); L. Wang and P.J. Steinhardt, Astrophys. J. 508, 483 (1998); R.R. Caldwell and P.J. Steinhardt, Phys. Rev. D 57, 6057 (1998); G. Huey, L. Wang, R. Dave, R.R. Caldwell, and P.J. Steinhardt, ibid. 59, 063005 (1999); P.F. González-Díaz, ibid. 62, 023513 (2000).

[9] P.J. Steinhardt, L. Wang, and I. Zlatev, Phys. Rev. Lett. 82, 896 
(1999); Phys. Rev. D 59, 123504 (1999); I. Zlatev and P.J. Steinhardt, Phys. Lett. B 459, 570 (1999).

[10] A.C. Baccigalupi, A. Balbi, S. Matarrase, F. Perrotta, and N. Vittorio, Phys. Rev. D 65, 063520 (2002); M. Melchiorri, L. Mersini, C.J. Odman, and M. Tradden, ibid. 68, 043509 (2003); M. Doupis, A. Riazuelo, Y. Zolnierowski, and A. Blanchard, Astron. Astrophys. 405, 409 (2003).

[11] R.R. Caldwell, M. Kamionkowski, and N.N. Weinberg, Phys. Rev. Lett. 91, 071301 (2003); G.W. Gibbons, hep-th/0302199; R.R. Caldwell, Phys. Lett. B 545, 23 (2002); Y.S. Piao and E. Zhou, Phys. Rev. D 68, 083515 (2003); Y.S. Piao and Y.Z. Zhang, astro-ph/0401231.

[12] A. Kamenshchik, U. Moschella, and V. Pasquier, Phys. Lett. B 511, 265 (2001); N. Bilic, G.B. Tupper, and R. Viollier, ibid. 535, 17 (2001); M.C. Bento, O. Bertolami, and A.A. Sen, Astrophys. J. 574, 538 (2002); J.S. Fabris, S.V. Gonzalves, and P.E. de Souza, Gen. Relativ. Gravit. 34, 53 (2002); V. Gorini, A. Kamenshchik, and U. Moschella, Phys. Rev. D 67, 063509 (2003); A. Dev, D. Jain, and J.E. Alcaniz, ibid. 67, 023515 (2003); J.D. Barrow, Phys. Lett. B 180, 335 (1986); 235, 40
(1990); D. Bazcia and J. Jackiw, Ann. Phys. (N.Y.) 270, 246 (1998); M. Makler, S. Quinet de Oliveira, and I. Waga, Phys. Lett. B 555, 1 (2003).

[13] P.F. González-Díaz, Phys. Lett. B 562, 1 (2003); Phys. Rev. D 68, 021303 (2003); Mod. Phys. Lett. A 18, 2599 (2003).

[14] J.S. Bagla, H.K. Jassal, and T. Padmanabhan, Phys. Rev. D 67, 063504 (2003).

[15] D. Bohm, Phys. Rev. 84, 166 (1951); 85, 180 (1952).

[16] Handbook of Mathematical Functions, edited by M. Abramowitz and I.A. Stegun (Dover, New York, 1965); I.S. Gradshteyn and I.M. Ryzhik, Table of Integrals, Series, and Products (Academic Press, Orlando, FL, 1980).

[17] T. Padmanabhan, Phys. Rev. D 66, 021301 (2002).

[18] T. Padmanabhan and T.R. Choudhury, Phys. Rev. D 66, 081301 (2002).

[19] P.J. Steinhardt, in Critical Problems in Physics, edited by V.L. Fitch and D.R. Marlow (Princeton University Press, Princeton, NJ, 1997); L.P. Chimento, A.S. Jakubi, and D. Pavon, Phys. Rev. D 67, 087302 (2003). 